\title{
Wireless water quality cloud monitoring system with self-healing algorithm
}

\begin{abstract}
The need of a water quality monitoring system is crucial for aquaculture and environmental control evaluation. This paper focuses on the development of the Water Quality (WQ) monitoring module that consists of hardware and software components. It highlights the details of the hardware components and the algorithm as well as the software that is connected to the cloud. There are many works on storing environmental data in cloud storage in Malaysia. The new platform to date for the Internet of Things (IoT) and cloud database is Favoriot. Favoriot is a platform for IoT and machine-to-machine (M2M) development. For this project, Favoriot platform is used for real time data. The self-healing algorithm is design to reduce human intervention and continuous data collected in the remote areas. The result shows that the self-healing algorithm is able to recover itself without physical reseting, in case during distruption of wireless service connection failure.
\end{abstract}

Keyword: Wireless water monitoring system; Cloud data storage; Favoriot; Self-healing algorithm 